\title{
Light radioactive nuclei capture reactions with phenomenological potential models.
}

\author{
V. Guimarães* and C. A Bertulani ${ }^{\dagger}$ \\ *Instituto de Física, Universidade de São Paulo, P.O. Box 66318, 05389-970 São Paulo, SP, Brazil \\ ${ }^{\dagger}$ Texas A\&M University-Commerce, Commerce, Texas 75429, USA.
}

\begin{abstract}
Light radioactive nuclei play an important role in many astrophysical environments. Due to very low cross sections of some neutron and proton capture reactions by these radioactive nuclei at energies of astrophysical interest, direct laboratory measurements are very difficult. For radioactive nuclei such as ${ }^{8} \mathrm{Li}$ and ${ }^{8} \mathrm{~B}$, the direct measurement of neutron capture reactions is impossible. Indirect methods have been applied to overcome these difficulties. In this work we will report on the results and discussion of phenomenological potential models used to determine some proton and neutron capture reactions. As a test we show the results for the ${ }^{16} \mathrm{O}(\mathrm{p}, \gamma)^{17} \mathrm{~F}_{g s}\left(5 / 2^{+}\right)$and ${ }^{16} \mathrm{O}(\mathrm{p}, \gamma){ }^{17} \mathrm{~F}_{e x}\left(1 / 2^{+}\right)$capture reactions. We also computed the nucleosynthesis cross sections for the ${ }^{7} \mathrm{Li}(\mathrm{n}, \gamma){ }^{8} \mathrm{Li}_{g s},{ }^{8} \mathrm{Li}(\mathrm{n}, \gamma){ }^{9} \mathrm{Li}_{g s}$ and ${ }^{8} \mathrm{~B}(\mathrm{p}, \gamma){ }^{9} \mathrm{C}_{g s}$ capture reactions.
\end{abstract}

Keywords: spectroscopy, cluster, elastic-transfer, radioactive beam.

PACS: $27.20 .+\mathrm{n}, 25.70 . \mathrm{Bc}, 21.10 . \mathrm{Jx}, 24.10 . \mathrm{Eq}, 25.60 . \mathrm{Je}$

\section{INTRODUCTION}

In the investigation of many astrophysical entities such as primordial universe, main path stellar evolution, novae, super-novae explosion, X-ray bursts etc. , the important input parameter in the models is the cross sections of the capture reactions as a function of energy. However, since in many of these environments the temperature is not very high, the cross sections have to be obtained at the "Gamow Peak" energies which are very low, in the range of few tens to at most hundreds of keVs. Whereas some of the cross sections originate from laboratory measurements, the majority are based on extrapolations from the higher to lower energies or are obtained by pure theoretical models with, sometimes, no firm experimental basis. Some reactions are practically impossible to be directly measured. For instance, for light radioactive nuclei nucleosynthesis, the neutron capture reaction is impossible because the combination of target+beam is not possible. A typical case is the ${ }^{8} \mathrm{Li}(\mathrm{n}, \gamma){ }^{9} \mathrm{Li}$ capture reaction where direct measurement is not possible because no ${ }^{8} \mathrm{Li}$ or neutron target exist. For proton capture reactions involving radioactive nuclei such as ${ }^{8} \mathrm{~B}$ or ${ }^{7} \mathrm{Be}$, the direct measurement is very difficult due to the low cross sections and limited beam intensities of these elements. For such cases, indirect methods to determine the cross sections were developed and are quite usually adopted. Extrapolation from higher energy data to lower regime is also not straight forward. Careful and accurate account of physically relevant information has to be considered in the description of the reaction before the extrapolation is performed, where to describe capture reactions not only information on the structure of the nuclei, but also a clear understand- 
ing of the reaction mechanism is required.

Many indirect methods have been developed to overcame the difficulties to obtain low energies cross sections. Among these methods we have the Coulomb dissociation method, which corresponds to the inverse temporal reaction of the capture [1], the trojan horse method [2], the reduced-width or ANC (Asymptotic Normalization Coefficient) method [3] and potential model, where the latter two use transfer reactions as a way to get information on the non-resonant part of the capture reaction process and will be discussed in more detail in the next section. These indirect methods are very suitable to be used in association with low-energy radioactive nuclear beams.

\section{LIGHT RADIOACTIVE NUCLEI AND NUCLEOSYNTHESIS.}

To overcame the $A=8$ gap and synthetize heavier elements the key nuclei are the light radioactive elements ${ }^{8} \mathrm{Li}(178 \mathrm{~ms})$ and ${ }^{8} \mathrm{~B}(770 \mathrm{~ms})$. These elements are important for instance in the inhomogeneous big-bang nucleosynthesis (IBBN) [4]. Because of the assumption of homogeneous baryon density distribution and also the instability of ${ }^{8} \mathrm{Be}$, the nuclear reaction flows stop at $A=8$ in SBBN (Standard Big Bang Nucleosynthesis). In IBBN the baryon density distribution is assumed to be inhomogeneous due to several cosmological processes before an onset of primordial nucleosynthesis. A difference in diffusion between protons and neutrons makes high density zones proton-rich and low density zones neutron-rich. In such an environment, heavy elements can be produced via the formation proton or neutron rich unstable nuclei. Also it is believed that in supermassive stars with low metalicity, the $3 \alpha$ capture process cannot form enough ${ }^{12} \mathrm{C}$ to initiate the rapid hydrogen burning during the explosion. However, in such high temperature and densities the hot $p p$-chain my bypass the slower $3 \alpha$ capture [5] and in this case ${ }^{8} \mathrm{~B}$ plays an important role.

The following nuclear reaction chains were found to be very important in nucleosynthesis processes discussed above to jump the $A=8$ gap and synthetise heavier elements:

chain (1): ${ }^{7} \operatorname{Li}(\mathrm{n}, \gamma){ }^{8} \mathrm{Li}(\alpha, \mathrm{n}){ }^{11} \mathrm{~B}(\mathrm{n}, \gamma){ }^{12} \mathrm{~B}\left(\mathrm{e}^{-} v\right){ }^{12} \mathrm{C}$.

chain (2): ${ }^{7} \mathrm{Li}(\mathrm{n}, \gamma){ }^{8} \mathrm{Li}(\mathrm{n}, \gamma){ }^{9} \mathrm{Li}\left(\mathrm{e}^{-} v\right){ }^{9} \mathrm{Be}(\mathrm{n}, \gamma){ }^{10} \mathrm{Be}\left(\mathrm{e}^{-} v\right){ }^{10} \mathrm{~B}(\mathrm{n}, \gamma){ }^{11} \mathrm{~B}(\mathrm{n}, \gamma){ }^{12} \mathrm{~B}\left(\mathrm{e}^{-} v\right){ }^{12} \mathrm{C}$.

chain (3): ${ }^{7} \mathrm{Be}(\mathrm{p}, \gamma){ }^{8} \mathrm{~B}(\alpha, \mathrm{p}){ }^{11} \mathrm{C}\left(\mathrm{e}^{+} v\right){ }^{11} \mathrm{~B}(\mathrm{p}, \gamma){ }^{12} \mathrm{C}$.

chain (4): ${ }^{7} \mathrm{Be}(\mathrm{p}, \gamma){ }^{8} \mathrm{~B}(\alpha, \mathrm{p}){ }^{11} \mathrm{C}(\mathrm{p}, \gamma){ }^{12} \mathrm{~N}(\beta){ }^{12} \mathrm{C}$.

chain (5): ${ }^{7} \mathrm{Be}(\mathrm{p}, \gamma){ }^{8} \mathrm{~B}(\mathrm{p}, \gamma){ }^{9} \mathrm{C}(\alpha, \mathrm{p})^{12} \mathrm{~N}(\beta){ }^{12} \mathrm{C}$.

The first two reaction chains play the central role in the production of neutron-rich nuclei, and the chains (3), (4) and (5) of proton-rich nuclei. The ${ }^{8} \mathrm{Li}(\mathrm{n}, \gamma){ }^{9} \mathrm{Li}$ is also an important reaction in the early stage of the ignition of supernovae, where two neutrons capture reactions, ${ }^{9} \mathrm{Li}(2 \mathrm{n}, \gamma){ }^{11} \mathrm{Li}$ and ${ }^{6} \mathrm{He}(2 \mathrm{n}, \gamma){ }^{8} \mathrm{He}(\beta)^{8} \mathrm{Li}$, may become important. The ${ }^{7} \mathrm{Be}(\mathrm{p}, \gamma){ }^{8} \mathrm{~B}$ is related to the solar neutrino problem. Precise predictions of the production rate of ${ }^{8} \mathrm{~B}$ solar neutrinos are important for testing solar models, and for limiting the allowed neutrino mixing parameters. This, however, it the most uncertain reaction leading to ${ }^{8} \mathrm{~B}$ formation in the Sun [6]. The ${ }^{8} \mathrm{~B}(\mathrm{p}, \gamma){ }^{9} \mathrm{C}$, capture reaction is also important in the novae environment where temperatures are several times larger than $10^{8} \mathrm{~K}$, 
corresponding to Gamow window energies around $E=50-300 \mathrm{keV}[5,7]$.

\section{$S$-FACTOR AT LOW ENERGIES}

Cross sections for many of the neutron and proton capture reactions by light radioactive nuclei are still poorly known or remain unmeasured at the required low energy region. For systems which cannot be measured directly at low energies some procedures or methods are adopted to obtain cross sections in this region. The most obvious way to obtain the low energy cross section is the extrapolation to low energy through polynomial parametrization of the available high energy region data. Actually these extrapolations are performed to the $S$-factor where the strong energy dependence of charged particle capture reactions due to the coulomb barrier penetration are removed. Thus, the cross sections are conventionally expressed in terms of the $S$-factor which is defined as:

$$
S(E)=E \sigma(E) \exp [2 \pi \eta(E)] \quad \text { with } \quad \eta(E)=Z_{a} Z_{b} e^{2} / \hbar v,
$$

where $v$ is the initial relative velocity between the nucleus and proton.

However, the $S$-factor does not remove completely the energy dependence, the structure of the final bound state, resonances and attenuation of the barrier by the nuclear mean field can give an extra energy dependency.

The problem is that the extrapolation is not usually a straight forward procedure and some physics has to be taken into account. For example, for some systems there is an upturn in the $S$-factor at very low energy due to a pole at threshold when the photon energy of a $A\left(x, E_{\gamma}\right) B$ capture reaction vanishes. This is a quite common feature and a straight line extrapolation of $S(E)$ from the high energy data region to $S(0)$ would not work. According to Jennings et al. [8], the presence of the pole suggests the $S$-factor may be parametrized as Laurent series $S=d_{1} \times E_{\gamma}^{-1}+d_{0}+d_{1} \times E_{\gamma}$, and they propose the following expression to parametrize the $S$-factor:

$$
\frac{S(E)}{S(0)}=\frac{a}{E_{B}+E}+b+c \times E \quad \text { or } \quad S(E)=\frac{A}{E_{B}+E}+B+C \times E
$$

A good test for this assumption is the investigation of the low energy region of the ${ }^{16} \mathrm{O}(\mathrm{p}, \gamma){ }^{17} \mathrm{~F}$ capture reaction. This reaction is an important reaction in the CNO cycle of our Sun but also in evolutionary phase referred as the asymptotic giant branch (AGB) of some massive stars [9]. A very good and precise set of data has been obtained for this reaction for both ground-state $\left(5 / 2^{+}\right)$and first excited state $\left(1 / 2^{+}\right)$transitions of ${ }^{17} \mathrm{~F}$, as can be seen in Figure 1. In the figure the upturn for the reaction leading to the $1 / 2^{+}$ excited state is very clear.

Let's consider only the data from Rolfs [11] of the transition to the ${ }^{17} \mathrm{~F}_{G S}(5 / 2+)$ ground-state. By fitting the data with the expression with pole effect and by a straight line we get the following parameters, respectively:

a) $S(E)=\frac{1.0574}{0.6+E}-0.4359+0.4584 \times E$, giving $S(0)=1.326$ and $\chi^{2}=0.194$

b) $S(E)=0.66189+0.1 \times E$, which gives $S(0)=0.66$ and $\chi^{2}=0.305$ 


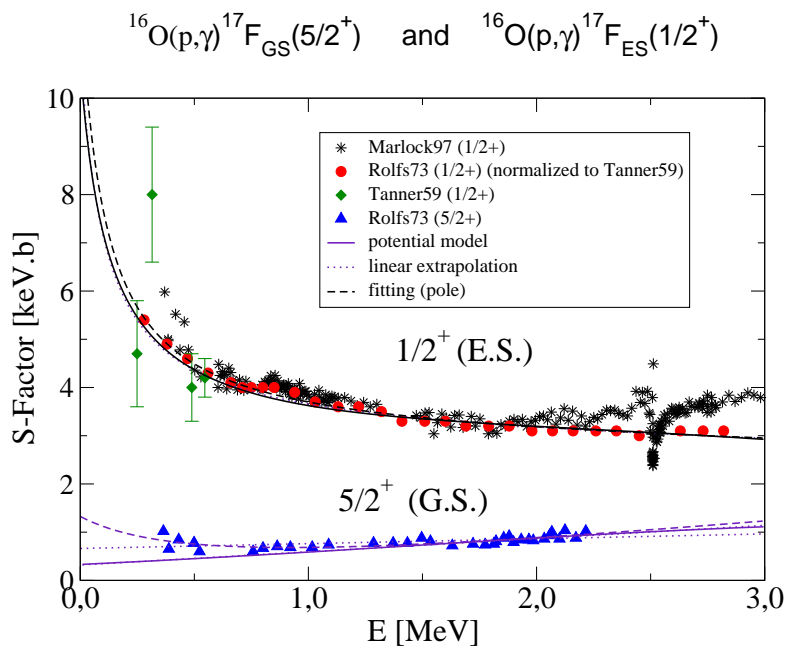

FIGURE 1. $S$-factor for the reaction ${ }^{16} \mathrm{O}(p, \gamma){ }^{17} \mathrm{~F}$. The experimental data are from Ref. $[10,11,12]$.

As we can see a very different value for $S(0)$ is obtained depending on the extrapolation performed. The solution for this problem is including more physics in the extrapolation to better determine the $S$-factor at low energies. This can be better done with another "fitting" method called R-matrix [13]. The idea of the R-Matrix calculations is to parameterize the cross sections with a small number of parameters and then used to extrapolate the cross section down to astrophysical energies. Since it deals with resonances in the continuum, it can be considered, to some extent, a generalization of the Breit-Wigner formalism. The R-matrix framework assumes that the space is divided into internal region (with radius match $a$ ), where nuclear forces are important, and the external region, where the interaction between the nuclei is governed by the Coulomb force only. For more details of this method we recommend the work of P. Descouvemont in ref. [13]. This method, however, has some limitations. It doesn't work for all systems, the radius match $a$ is not clearly determined (usually it is arbitrary chosen) and it is valid only for low level density composite nuclei. Moreover, it has adjusted parameters which not always has a clear physical meaning.

Other possibility is to use what we call "non-Fitting" methods. These other methods are related to theoretical calculations and they determine the cross sections from wave functions with, in principle, no data is required. Among these methods we have the microscopic cluster models and Microscopic "ab initio" models. In the first, a manybody Hamiltonian with embedded cluster are involved in a more complex calculations. Internal structure has to be taken into account and the resonating group method can be applied. In the second, even more complicate calculations are involved since continuum states, which are very difficult to take into account, have to be considered. Applications of these two calculations to the cases of ${ }^{7} \mathrm{Li}(\mathrm{n}, \gamma){ }^{8} \mathrm{Li}$ and ${ }^{7} \mathrm{Be}(\mathrm{p}, \gamma){ }^{8} \mathrm{~B}$ capture reactions can be seen in ref. [14]. Another method also considered as "non - Fitting" method is the Potential model, where ANC (Asymptotic Normalization Coefficient) can be considered 
as an approximation. This method is quite simple to solve numerically since no structure of the colliding nuclei has to be taken into account.

\section{THE POTENTIAL MODEL}

In the potential model, the direct radiative capture (DRC) of an $s$ - and/or $d$-wave nucleon (proton or neutron) by a nucleus $A$, proceeding via E1 transition and leaving the compound nucleus $B$ in its ground state, is given by:

$$
\sigma_{A \rightarrow B}^{E 1}(n, \gamma)=\frac{16 \pi}{9 \hbar} k_{\gamma}^{3}\left|<\Psi_{\text {scat }}\right| O^{E 1}\left|\Psi_{\text {bound }}>\right|^{2},
$$

where $k_{\gamma}=\varepsilon_{\gamma} / \hbar c$ is the wave number corresponding to a $\gamma$-ray energy $\varepsilon_{\gamma}, O^{E 1}$ stands for the electric dipole operator, the initial-state wave function $\Psi_{s c a t}$ is the incoming nucleon wave function in the nucleon-nucleus potential and $\Psi_{\text {bound }}$ is wave function which describes the single-particle bound state.

Although the potential model works well for many nuclear reactions of interest in astrophysics, it is often necessary to pursue a more microscopic approach to reproduce experimental data. In a microscopic approach, instead of the single-particle wave functions one often makes use of overlap integrals, $I_{\text {bound }}(\mathbf{r})$, and a many-body wave function for the relative motion, $\Psi_{\text {scat }}(\mathbf{r})$. The effect of many-body will eventually disappear at large distances between the nucleon and the nucleus. One thus expects that the overlap function asymptotically matches the solution of the Schrödinger equation, with $V=V_{\text {Coul }}$ (Pure Coulomb) for protons and $V=0$ for neutrons. This assumption may be true only for a very peripheral capture reaction. This approximation, when $r \rightarrow \infty$, is called ANC (Asymptotic Normalization Coefficient) and:

$$
I_{\text {bound }}(r)=A N C \times \frac{W_{-\eta, l_{b}+1 / 2}(2 \kappa r)}{r} \quad \text { (for protons) }
$$

where the binding energy of the $A+x$ system is related to $\kappa$ by means of $E_{B}=$ $\hbar^{2} \kappa^{2} / 2 m_{n x}, W_{p, q}$ is the Whittaker function and ANC is the asymptotic normalization coefficient. The $A N C$ can be obtained from peripheral transfer reactions whose amplitudes contain the same overlap function as the amplitude of the corresponding capture reaction of interest [3].

In terms of overlap integral the direct capture cross sections are obtained from the calculation of

$$
\sigma_{L, J_{b}}^{\text {d.c. }} \propto\left|<I_{\text {bound }}(r) \| r^{L} Y_{L}\right|\left|\Psi_{\text {scat }}(r)>\right|^{2} .
$$

However, it has been shown that $s$-wave neutron capture, even at rather low energies, is not peripheral $[15,16]$ and so it is necessary to calculate the wave function of the incoming neutron or proton and the wave function for the bound system. Thus, in the potential model, it is necessary to calculate the overlap function also taking into account the internal part of the nuclear potential, $\mathrm{I}_{\text {bound }}=S F^{1 / 2} \times \Psi(r)_{\text {bound }}$. Here, $S F^{1 / 2}$ is the 
spectroscopic amplitude and $\Psi(r)_{\text {bound }}$ is the wave-function which describes the bound state.

In the potential model, the continuum wave function, $\Psi_{\text {scat }}$, has to be calculated with a potential which includes also the nuclear interaction. Thus, the essential ingredients in the potential model are the potentials used to generate the wave functions $\Psi_{\text {scat }}$ and $I_{\text {bound }}$, and the normalization for the latter which is given by its spectroscopic factor. Here we use a Woods-Saxon (WS) parameterization to build up the potentials $V_{0}(r)$ and $V_{S O}(r)$, where for the latter we consider the derivative of the WS form factor. The parameters $V_{0}, V_{S 0}, R_{0}, a_{0}, R_{S 0}$, and $a_{S 0}$ are chosen to reproduce the ground state energy $E_{B}$ (or the energy of an excited state). For this purpose, we define typical values (Table I) for $V_{S 0}, R_{0}, a_{0}, R_{S 0}$, and vary only the depth of the central potential, $V_{0}$. A different set of potential depths might be used for continuum states. To calculate the non-resonant part of these capture reactions in the framework of the potential model we used the computer code RADCAP developed by Bertulani [17].

We have tested this potential model by determining the ${ }^{16} \mathrm{O}(\mathrm{p}, \gamma){ }^{17} \mathrm{~F}_{g s}$, and ${ }^{16} \mathrm{O}(\mathrm{p}, \gamma){ }^{17} \mathrm{~F}_{1 \text { st }} S$-factor as a function of energy. In Table-I we list all the parameters of the potentials used to generate the incoming and bound wave functions. All the potentials were assumed to be a Woods-Saxon shape with geometric parameters $r_{0}=1.25$ and $a=0.65 \mathrm{fm}$. The $J_{B}=5 / 2^{+}$ground state $\left(J_{B}=1 / 2^{+}\right.$excited state $)$of ${ }^{17} \mathrm{~F}$ is described as a $j_{B}=d_{5 / 2}$ proton $\left(j_{B}=s_{1 / 2}\right.$ proton $)$ coupled to the ${ }^{16} \mathrm{O}$ core, which has an intrinsic spin $I_{A}=0^{+}$. The gamma-ray transitions are dominated by the $E 1$ multipolarity and by incoming $p$ waves for both states. The M1 and E2 contributions amount to less than $0.1 \%$ of the dominant E1 contribution, as shown in Ref. [11] where a potential model was also used. The spin-orbit potential depth $-10.0 \mathrm{MeV}$ and the spectroscopic factor 1.0 have been used for both states, although 0.9 and 1.0 for the ground state and the excited state, respectively, are recommended by Rolfs in Ref. [11]. On the other hand, Iliadis et al. [18] recommend values close to unity. The continuum states potential depth are set as the same as that of bound states, since no elastic scattering data is known for this system. Our results are shown in Figure 2 with solid line, and as we can see, it reproduces quite well the experimental data. The parameterization of the potential model calculations for the ${ }^{16} \mathrm{O}(\mathrm{p}, \gamma){ }^{17} \mathrm{~F}$ capture reactions with the pole expression is given by:

for ${ }^{17} \mathrm{~F}_{G S}(5 / 2+): S(E)=\frac{0.0011578}{0.6+E}+0.3207+0.2734 \times E$, giving $\mathrm{S}(0)=0.319$ and $\chi^{2}=0.0139$;

for ${ }^{17} \mathrm{~F}_{E S}(1 / 2+): S(E)=\frac{0.8826}{0.11+E}+2.9640-0.1005 \times E$, with $\mathrm{S}(0)=10.987$ and $\chi^{2}=0.2019$

Here also we present the results for the ${ }^{7} \mathrm{Li}(\mathrm{n}, \gamma){ }^{8} \mathrm{Li}_{g s, 1 s t},{ }^{8} \mathrm{Li}(\mathrm{n}, \gamma){ }^{9} \mathrm{Li}_{g s},{ }^{7} \mathrm{Be}(\mathrm{p}, \gamma){ }^{8} \mathrm{~B}_{g s}$ and ${ }^{8} \mathrm{~B}(\mathrm{p}, \gamma){ }^{9} \mathrm{C}_{g s}$

For the ${ }^{7} \mathrm{Li}(\mathrm{n}, \gamma){ }^{8} \mathrm{Li}_{g s, 1 s t}$ capture reaction, the gamma-ray transitions are dominated by the $E 1$ multipolarity and by incoming $s$ waves and $d$ waves. The $J_{B}=2^{+}$ground state $\left(J_{B}=1^{+}\right.$first excited state) of ${ }^{8} \mathrm{Li}$ is described as a $j_{B}=p_{3 / 2}$ neutron interacting with the ${ }^{7} \mathrm{Li}$ core, which has an intrinsic spin $I_{x}=3 / 2^{-}$. Here we used $R_{0}=R_{C}=R_{S 0}=2.391$ 
${ }^{7} \operatorname{Li}(\mathrm{n}, \gamma)^{8} \mathrm{Li}_{\mathrm{GS}(2+)+1 \mathrm{st}(1+)}$

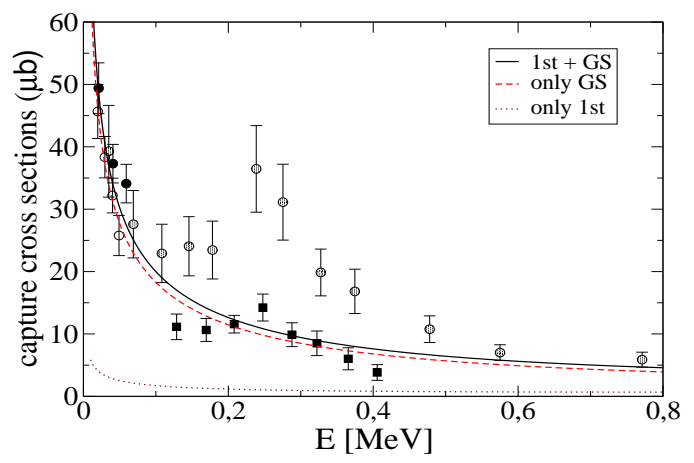

FIGURE 2. Potential model calculations for the reaction ${ }^{7} \mathrm{Li}(\mathrm{n}, \gamma)^{8} \mathrm{Li}$. Experimental data are from Refs. $[16,19,20,21,22]$.

fm for the radius parameters. For the continuum state, the potential depth was obtained from the analysis of Nagai [16] which was determined from the experimental scattering lengths, see Table-I for the values. The spectroscopic factors adopted where $S F($ g.s. $)=$ 0.98 [23] and $S F(1 s t)=0.48$ [16] for the ground and first excited states, respectively. The capture to the first excited state contributes to less than $5 \%$ of the total cross section. The results for this calculation are shown in Figure 2.

For the ${ }^{8} \mathrm{Li}(\mathrm{n}, \gamma){ }^{9} \mathrm{Li}_{g s}$ capture reaction, the scattering potential parameters for both entrance channel spins, $s=5 / 2^{+}, 3 / 2^{+}$, for the ${ }^{8} \operatorname{Li}\left(2^{+}\right)+n$ system were obtained by keeping the same volume integral per nucleon, $\mathrm{J}_{V} / \mathrm{A}$, as those for the entrance channel spins, $s=2^{+}, 1^{+}$, deduced from the scattering potentials of the ${ }^{7} \mathrm{Li}+\mathrm{n}$ system [16]. The results are presented in Figure 4. Details of the analysis for this neutron capture reactions ${ }^{7} \mathrm{Li}(\mathrm{n}, \gamma){ }^{8} \mathrm{Li}_{g s, 1 s t}$ and ${ }^{8} \mathrm{Li}(\mathrm{n}, \gamma){ }^{9} \mathrm{Li}_{g s}$ are published in Ref. [23].

For the ${ }^{7} \mathrm{Be}(\mathrm{p}, \gamma){ }^{8} \mathrm{~B}_{g s}$ capture reaction, the $J_{B}=2^{+}$ground state of ${ }^{8} \mathrm{~B}$ is described as a $j_{B}=p_{3 / 2}$ proton coupled to the ${ }^{7} \mathrm{Be}$ core, which has an intrinsic spin $I_{A}=3 / 2^{-}$. For this system we adopted $a=0.52 \mathrm{fm}$ and $V_{s o}=-9.8 \mathrm{MeV}$. This is the same set of values adopted in Ref. [17]. The gamma-ray transition is dominated by the $E 1$ multipolarity and by incoming $s$ and $d$ waves. Our results are shown in Figure 3. To reproduce the M1 resonance we considered $V_{S C A T}=-38.14 \mathrm{MeV}$ and $S F=0.7$ (dashed-dotted line) also with the other parameters according to Table I.

For the ${ }^{8} \mathrm{~B}(\mathrm{p}, \gamma){ }^{9} \mathrm{C}_{g s}$ no data is available. The capture process for this reaction is dominated by E1 transitions from incoming $s$-waves to bound $p$ states in the ground state of ${ }^{9} \mathrm{C}\left(J_{B}=3 / 2^{-}\right)$, which is described as a $j_{B}=p_{3 / 2}$ proton coupled to the ${ }^{8} \mathrm{~B}$ core, which has an intrinsic spin $I_{A}=2^{+}$. The spectroscopic factor has been set to 1.0 as in Ref. [30], where several spectroscopic factor values are compared. The results are shown in Figure-4.

\section{FINAL REMARKS}




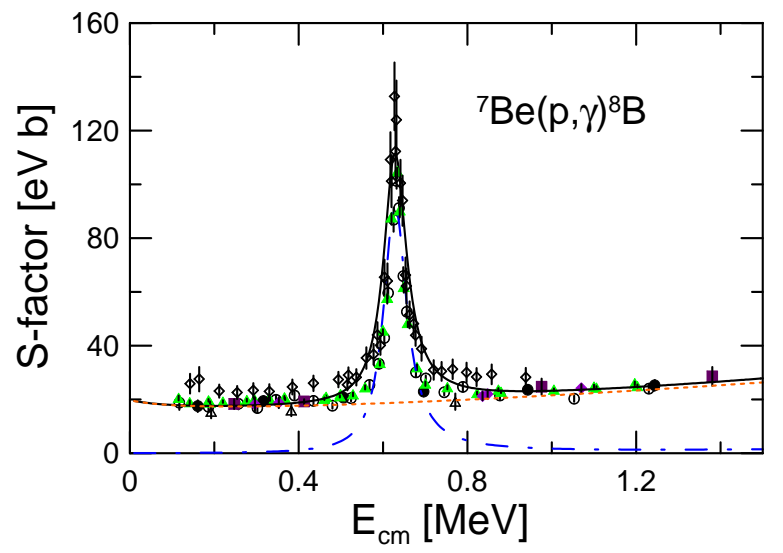

FIGURE 3. (Potential model calculations for the reaction ${ }^{7} \mathrm{Be}(\mathrm{p}, \gamma)^{8} \mathrm{~B}$. The dashed-dotted line is the calculation for the $\mathrm{M} 1$ resonance at $E_{c m}=0.63 \mathrm{MeV}$ and the dotted line is for the non-resonant capture. Experimental data are from Refs. [24, 25, 26, 6, 27, 28]. The total S factor is shown as a solid line.
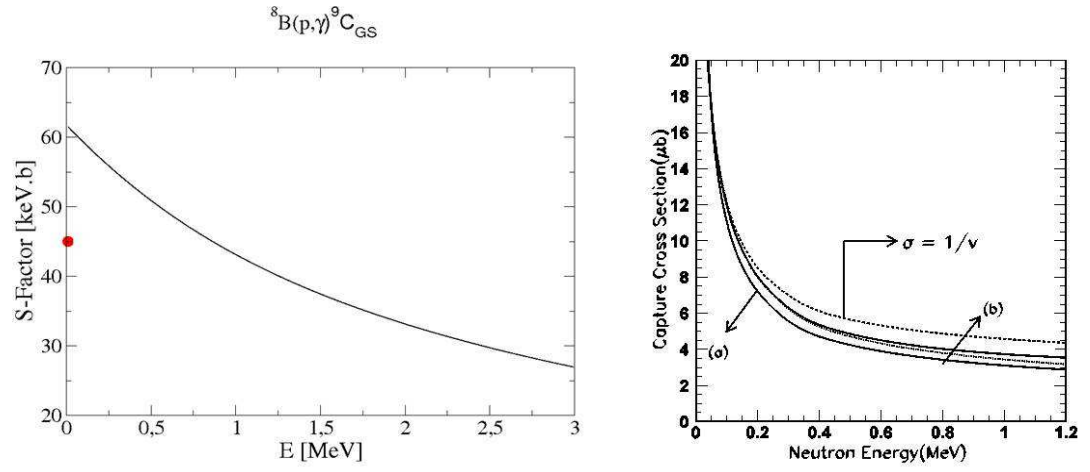

FIGURE 4. (a) Potential model calculations for the reaction ${ }^{8} \mathrm{~B}(\mathrm{p}, \gamma){ }^{9} \mathrm{C}$. The solid circle symbol at $E=0$ is from Refs. [29], which were obtained by ANC (extracted from breakup reaction) calculation. (b) Potential model calculations for the ${ }^{8} \mathrm{Li}(\mathrm{n}, \gamma){ }^{9} \mathrm{Li}$ capture reaction. The lower curves labeled (a) correspond to the adoption of scattering potential derived from Table-I. Curves labeled (b) correspond to the assumption of same potential for the incoming wave function as for the bound state, for s-wave neutron only (dotted curve) and s- and d-wave neutrons (solid curve). The uppermost curve (dashed line) corresponds to a 1/v fit to the low-energy cross sections.

In this work, we report the results obtained for the non-resonant part of the neutron and proton capture reactions of light nuclei; ${ }^{7} \mathrm{Li}(\mathrm{n}, \gamma){ }^{8} \mathrm{Li},{ }^{8} \mathrm{Li}(\mathrm{n}, \gamma){ }^{9} \mathrm{Li},{ }^{7} \mathrm{Be}(\mathrm{p}, \gamma){ }^{8} \mathrm{~B}$ and ${ }^{8} \mathrm{~B}(\mathrm{p}, \gamma){ }^{9} \mathrm{C}$ in the framework of the potential model. As a test of the model we show the results for the ${ }^{16} \mathrm{O}(\mathrm{p}, \gamma){ }^{17} \mathrm{~F}_{g s}\left(5 / 2^{+}\right)$and ${ }^{16} \mathrm{O}(\mathrm{p}, \gamma){ }^{17} \mathrm{~F}_{e x}(1 / 2+)$ capture reactions. These calculations are part of a more extensive series of calculations of neutron and proton capture reactions by light radioactive nuclei published elsewhere [31].

Although we have obtained the incoming nucleon scattering potentials for the reaction of interest from analysis of close systems, or by considering the same as the bound state, it would be interesting to obtain such potentials from direct elastic scattering measurement as ${ }^{8} \mathrm{Li}+\mathrm{p}$ and ${ }^{8} \mathrm{~B}+\mathrm{p}$. A program of investigation for these elastic scattering 
TABLE 1. Wood-Saxon potential parameters used in the calculations. Depths and B.E. are in $\mathrm{MeV}$ with $r_{0}=r_{S O}=r_{\text {coul }}=1.25 \mathrm{fm}$ and $a=a_{S O}=0.65 \mathrm{fm}$, where the radii are given by $R=r_{0} \times A_{T}^{1 / 3}$. The spin-orbit is given by $V_{S O}=-10 \mathrm{MeV}$.

\begin{tabular}{|cccccc|}
\hline & B.E. & $\mathrm{V}_{0}$ (bound) & SF & channel spin & $\mathrm{V}_{0}$ (scat) \\
\hline${ }^{16} \mathrm{O}+\mathrm{p}={ }^{17} \mathrm{~F}_{g s}\left(5 / 2^{+}\right)$ & 0.60 & 51.79 & 1.0 & & 51.79 \\
${ }^{16} \mathrm{O}+\mathrm{p}={ }^{17} \mathrm{~F}_{1 s t}\left(1 / 2^{+}\right.$ & 0.11 & 51.42 & 1.0 & & 51.42 \\
${ }^{7} \mathrm{Be}+\mathrm{p}={ }^{8} \mathrm{~B}_{g s}\left(2^{+}\right)$ & 0.14 & 41.26 & 1.0 & & 41.26 \\
${ }^{8} \mathrm{~B}+\mathrm{p}={ }^{9} \mathrm{C}_{g s}\left(3 / 2^{-}\right)$ & 1.30 & 41.97 & 1.0 & & 41.97 \\
${ }^{7} \mathrm{Li}+\mathrm{n}={ }^{8} \mathrm{Li}_{g s}\left(2^{+}\right)$ & 2.03 & 46.38 & $0.98(15)$ & $2^{+}, 1^{+}$ & $56.15,46.50$ \\
${ }^{7} \mathrm{Li}+\mathrm{n}={ }^{8} \mathrm{Li}_{1 s t}\left(1^{+}\right)$ & 1.05 & 43.30 & 0.48 & $2^{+}, 1^{+}$ & $56.15,46.50$ \\
${ }^{8} \mathrm{Li}+\mathrm{n}={ }^{9} \mathrm{Li}_{g s}\left(3 / 2^{-}\right)$ & 4.06 & 47.82 & $0.62(13)$ & $5 / 2^{+}, 3 / 2^{+}$ & $58.15,48.15$ \\
\hline
\end{tabular}

experiments at low energy is under way at the Sao Paulo University using the radioactive ion beam facility RIBRAS [32].

\section{REFERENCES}

1. G. Baur C. A. Bertulani and H. Rebel, Nucl. Phys. A 458, 188 (1986).

2. C. Spitaleri, et al., Nuclear Physics A 719, 99c-106c (2003).

3. C.A.Gagliardi, et al., Eur. Phys. J. A13, 227 (2002).

4. R. A. Malaney and W. A. Fowler, Astrophys. J. 333 (1989) 14.

5. M. Wiescher et al., Astrophys. J. 343, 352 (1989).

6. A. R. Junghans et al, Phys. Rev. C 68, 065803 (2003).

7. G.M. Fuller, S.E. Woosley, and T.A. Weaver, Astrophys. J. 307, 675 (1986).

8. B. K. Jennings, S. Karataglidis, T. D. Shoppa, Phys. Rev. C 58 (1998) 3711.

9. F. Herwig, Annu. Rev. Astron. Astrophys. 43, 435 (2005).

10. N. Tanner, Phys. Rev. 1141060 (1959).

11. C. Rolfs, Nuclear Physics A217 (1973) 29-70;

12. R. Morlock, R. Kunz, A. Mayer et al, Phys. Rev. Lett. 79, 3837 (1997).

13. P. Descouvemont et al., Atomic Data and Nuclear Data Tables 88, 203-236 (2004).

14. P. Descouvemont, Astrophys. J. Lett. 405, 518 (1993).

15. A. Mengoni, T. Otsuka, and M. Ishihara, Phys. Rev. C 52, R2334 (1995).

16. Y. Nagai, et al., Phys. Rev. C 71, 055803 (2005).

17. C. A. Bertulani, Comput. Phys. Commun. 156 (2003) 123.

18. C. Iliadis, C. Angulo, P. Descouvemont, M. Lugaro and P. Mohr, Phys. Rev. C 77 (2008) 045802.

19. M. Heil, F. Kaeppeler, M.Wiescher, and A. Mengoni,Astrophys. J. 507, 997 (1998).

20. Y. Nagai, et al. Astrophys. J. 381, 444 (1991).

21. M. Wiescher, R. Steininger, and F. Kappeler, Astrophys. J. 344, 464 (1989).

22. W. L. Imhof et al., Phys. Rev. 114, 1037 (1959).

23. V. Guimarães et al, Phys. Rev. C 75 (2007) 054602.

24. F. J. Vaughn, R. A. Chalmers, D. Kohler and L. F. Chase, Phys. Rev. C 2, 1657 (1970).

25. B. W. Filippone, A. J. Elwyn, C. N. Davids and D. D. Koetke, Phys. Rev.C 28, 2222 (1983).

26. L. T. Baby et al., Phys. Rev. Lett. 90, 022501 (2003).

27. N. Iwasa et al., Phys. Rev. Lett. 83, 2910 (1999).

28. R.W. Kavanagh et al., Bull. Am. Phys. Soc. 14, 1209 (1969).

29. L. Trache et al, Phys. Rev. C 66, 035801 (2002).

30. P. Mohr, Phys. Rev. C 67, 065802 (2003).

31. J. T. Huang, C. A. Bertulani, V. Guimaraes, At. Data and Nucl. Data Table, accepted (2010).

32. R. Lichtenthaler, A. Lepine-Szily, V. Guimaraes, et al., Eur. Phys. J. 25, 733 (2005) 